\title{
«Medizin, aber nicht nur»
}

\section{Matthias Scholer}

Wissenschaftsjournalist

Hermann Amstad war während 18 Jahren für die Schweizerische Akademie der Medizinischen Wissenschaften (SAMW) tätig. Dabei leitete der Mediziner während der letzten elf Jahre als Generalsekretär die Geschäftsstelle. Auf Ende 2017 trat Hermann Amstad zurück. Eine gute Gelegenheit für einen Rückblick und spannende Einblicke in eine Institution, bei der die Autonomie des Einzelnen und der Dialog mit den vielfältigen Anspruchsgruppen ganz oben auf der Werteskala stehen.

Herr Amstad, Sie haben einen etwas ungewöhnlichen Lebenslauf. Nach dem Medizinstudium folgten verschiedene Tätigkeiten im Gesundheitsbereich, aber keine in der ärztlichen Praxis ...

Tatsächlich entspricht mein Werdegang meinen schon früh formulierten Zielvorstellungen. So gab ich in der Maturabroschüre am Kollegium Engelberg als Berufswunsch «Medizin, aber nicht nur» an. Nun, 40 Jahre später, steht fest, dass ich diese Vorgabe erreicht habe.

Sie kennen nach fast zwei Jahrzehnten die SAMW so gut wie kaum jemand. Die SAMW ist eine Forschungsförderungsinstitution. Was muss man sich unter diesem Begriff vorstellen?

Im Schweizer Forschungsförderungsgesetz werden zwei Hauptinstitutionen unterschieden: einerseits der Schweizerische Nationalfonds SNF, der Forschungs-

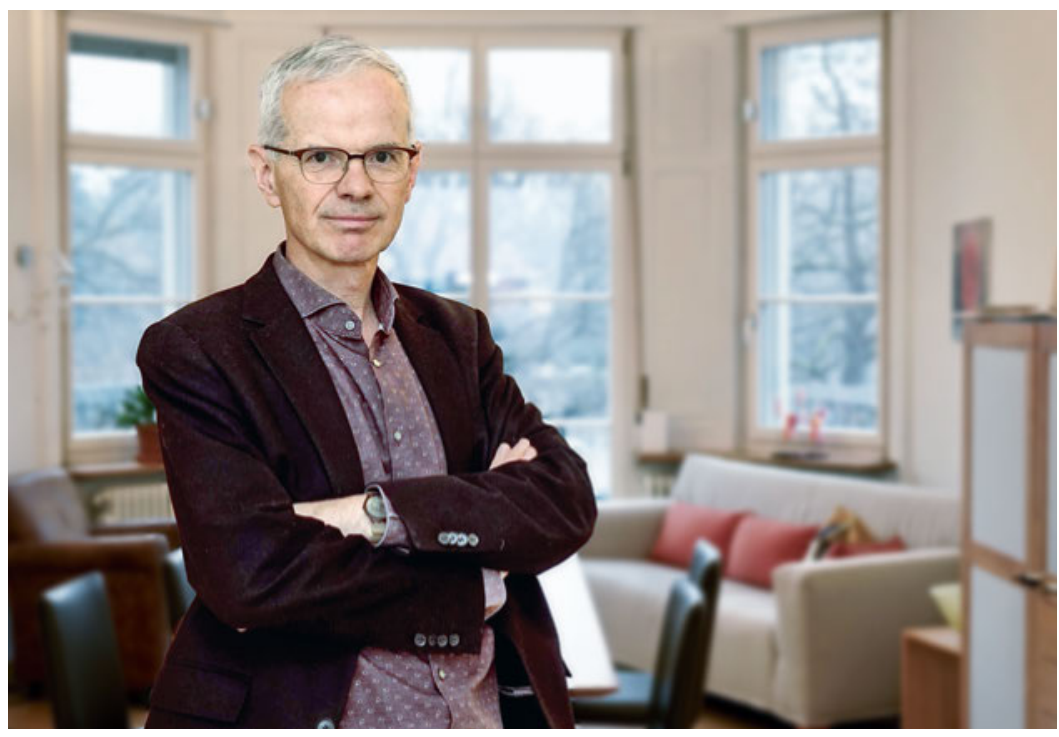

Hermann Amstad leitete während über zehn Jahren die Geschäftsstelle der SAMW. projekte mit Geld unterstützt, andererseits die Akademien der Wissenschaften, zu denen auch die SAMW gehört. Die Akademien haben drei Hauptaufgaben: Sie betreiben und fördern die Früherkennung gesellschaftlich relevanter Themen in den Bereichen Bildung, Forschung und Innovation; sie setzen sich zweitens dafür ein, dass die Forschung und die Forschenden ihre ethische Verantwortung wahrnehmen; und sie gestalten drittens den Dialog zwischen Wissenschaft und Gesellschaft.

Wie muss man sich diesen Dialog mit der Gesellschaft vorstellen? Macht die SAMW Umfragen zu konkreten Gesundheitsthemen?

Nein, das nicht. In den Gremien der SAMW sind jedoch Persönlichkeiten aus ganz unterschiedlichen Bereichen - nicht nur der Medizin - tätig; dadurch verfügt die Institution über ein gutes Sensorium für gesellschaftlich relevante Themen.

\section{Wie finanziert sich die SAMW?}

Das Gros der Finanzierung erfolgt über Bundesgelder. Diese erlauben der Akademie, Fragestellungen unabhängig von etwaigen Stakeholdern aufzuarbeiten. Zudem kann die SAMW auch auf Gelder aus Legaten und von privaten Stiftungen zurückgreifen. Diese sind jedoch meist thematisch gebunden.

\section{Die SAMW ist auch bekannt für ihre ethischen} Richtlinien. Wie werden solche erarbeitet?

Dafür gibt es ein klar festgelegtes Verfahren. Zuerst wird im Auftrag der Zentralen Ethikkommission (ZEK) der SAMW eine Arbeitsgruppe eingesetzt, welche einen ersten Entwurf ausarbeitet. Bereits im Entstehungsprozess führt die Arbeitsgruppe Expertenhearings durch und holt die Rückmeldung von ausgewählten 
Stakeholdern ein. Die ZEK begutachtet den Richtlinienentwurf fachlich, und der Vorstand der SAMW prüft ihn auf politische Opportunität. Der Senat, das oberste Gremium der SAMW, gibt den Richtlinienentwurf anschliessend $\mathrm{zu}$ einer dreimonatigen öffentlichen Vernehmlassung frei. Während dieser Zeit werden die für eine bestimmte Thematik relevanten Institutionen und Organisationen explizit eingeladen, sich zum Entwurf zu äussern. Die zuständige Arbeitsgruppe prüft

Bei komplexen Themen braucht es zwingend eine unabhängige Instanz wie die SAMW, um neue Denkanstösse und Ansätze für eine Trendwende finden zu können.

alle eingegangenen Stellungnahmen und erstellt eine finale Version. Diese wird nach erneuter Prüfung durch ZEK und Vorstand schlussendlich vom Senat verabschiedet. Dieser mehrstufige Ablauf garantiert einen hohen Grad an Unabhängigkeit.

\section{Sie haben von Arbeitsgruppen gesprochen. Wie setzen sich diese zusammen?}

Die Arbeitsgruppen sind interprofessionell zusammengesetzt. In der Regel arbeiten darin Mediziner, Pflegende, Ethikerinnen, Juristen und Vertreter von Patientenorganisationen zusammen. Zudem wird auf eine ausgewogene Verteilung der Geschlechter und der regionalen Herkunft geachtet.

\section{"Nachhaltiges Gesundheitssystem" war eines} der grossen Projekte während Ihrer Zeit als Generalsekretär. Ein äusserst komplexes Thema ...

Und ein Thema, bei dem viele Stakeholder involviert sind. Bei komplexen Themen braucht es zwingend eine unabhängige Instanz wie die SAMW, um neue Denkanstösse und Ansätze für eine Trendwende finden zu können.

\section{Können Sie uns ein Beispiel für einen solchen Ansatz nennen?}

Wenn man zum Beispiel weiss, dass auch die Bildung und eine gute Infrastruktur einen grossen Einfluss auf die Gesundheit einer Bevölkerung haben, dann macht es doch langfristig keinen Sinn, immer mehr Geld in das Gesundheitssystem zu pumpen, vor allem wenn dies auf Kosten der erwähnten Bereiche geht.

\section{Ende 2017 hat die SAMW die neuen Richtlinien "Umgang mit Sterben und Tod" in die Vernehm- lassung gegeben. Ein Thema, mit dem sich die SAMW regelmässig befasst ...}

Ja, und ein gutes Beispiel dafür, wie die SAMW auf gesellschaftliche Entwicklungen reagiert. Die ersten Richtlinien zum Thema Sterbehilfe wurden in den 1970er Jahren veröffentlicht. Damals wurde die Suizidhilfe als Teil der ärztlichen Tätigkeit klar abgelehnt. Doch der Wunsch, den eigenen Todeszeitpunkt selber bestimmen zu können, wurde immer lauter. Heute, fast 50 Jahre später, ist die Lebensqualität eines Patienten für die meisten Ärztinnen und Ärzte eine wichtigere Zielgrösse als eine Lebensverlängerung um jeden Preis. In solchen Spannungsfeldern müssen auch die Richtlinien konstant überprüft werden. Sie sollen Leitlinien geben in diesem heiklen Bereich, aber auch zwischen den nach wie vor sehr unterschiedlichen Sichtweisen und Wertvorstellungen vermitteln. Insbesondere sollen sie darauf hinwirken, dass die Selbstbestimmung aller Beteiligten - der Patientinnen, der Angehörigen und der medizinischen Fachpersonen geachtet und geschützt wird.

\section{Für Sie beginnt nun ein neuer Lebensabschnitt.} Bleiben Sie Ihrem Motto «Medizin, aber nicht nur» treu?

Ja, daran ändert sich nichts. Ich werde künftig einerseits als Senior Consultant meine Erfahrungen an verschiedenen Stellen im Gesundheitswesen einbringen und andererseits vermehrt lesen, malen und wandern.

Bildnachweis

(c) Matthias Scholer

Kurzer Lebenslauf Hermann Amstad

- Medizinstudium in Basel

- Medizinischer Lektor der «Schweizerischen Medizinischen Wochenschrift”

- Wissenschaftlicher Mitarbeiter schulärztlicher Dienst, Basel

- Master in Public Health

- Wissenschaftlicher Mitarbeiter SAMW (1999-2006)

- Generalsekretär SAMW (2007-2017)

- Senior Consultant für Gesundheitsorganisationen (amstad-kor.ch) 